\title{
Treadmill exercise ameliorates social isolation-induced depression through neuronal generation in rat pups
}

\author{
Jung-Wan Cho', Sun-Young Jung ${ }^{2}$, Sang-Won Lee ${ }^{3}$, Sam-Jun Lee ${ }^{4}$, Tae-Beom Seo ${ }^{5}$, Young-Pyo Kim ${ }^{5}$, Dae-Young Kim,* \\ 'Department of Physiology, College of Medicine, Kyung Hee University, Seoul, Korea \\ 2Department of Physical Therapy, Hosan University, Gyeongsan, Korea \\ ${ }^{3}$ Department of Physical Education, Korea Military Academy, Seoul, Korea \\ ${ }^{4}$ Department of Physical Education, College of Health, Welfare, and Education, Tong Myong University, Busan, Korea \\ ${ }^{5}$ Department of Kinesiology, College of Natural Science, Jeju National University, Jeju, Korea \\ ${ }^{6}$ Department of Sports Healthcare, College of Humanities \& Social Sciences, Inje University, Gimhae, Korea
}

Social isolation is known to induce emotional and behavioral changes in animals and humans. The effect of treadmill exercise on depression was investigated using social isolated rat pups. The rat pups in the social isolation groups were housed individually. The rat pups in the exercise groups were forced to run on treadmill for 30 min once a day from postnatal day 21 to postnatal day 34 . In order to evaluate depression state of rat pups, forced swimming test was performed. Newly generated cells in the hippocampal dentate gyrus were determined by 5-bromo-2'-deoxyuridine (BrdU) immunohistochemistry. We examined the expression of 5-hydroxytryptamine (5-HT) and tryptophan hydroxylase (TPH) in the dorsal raphe using immunofluorescence. The expression of brain-derived neurotrophic factor (BDNF) and tyrosine kinase B (TrkB) was detected by Western blot analysis. The present results demon- strated that social isolation increased resting time and decreased mobility time. Expression of 5-HT and TPH in the dorsal raphe and expression of BDNF and TrkB in the hippocampus were decreased by social isolation. The number of BrdU-positive cells in the hippocampal dentate gyrus was suppressed by social isolation. Treadmill exercise decreased resting time and increased mobility in the social isolated rat pups. Expression of $5-\mathrm{HT}, \mathrm{TPH}, \mathrm{BDNF}$, and TrkB was increased by treadmill exercise. The present results suggested that treadmill exercise may ameliorates social isolation-induced depression through increasing neuronal generation.

Keywords: Social isolation, Treadmill exercise, Depression, Neuronal generation

\section{INTRODUCTION}

Social isolation is known to induce emotional and behavioral changes in animals and humans (Fone and Porkess, 2008). Social isolation resulted in aggressiveness and depression-like behaviors in rats (Dandekar et al., 2009). Neurochemical imbalance in the brain regions contributes pathogenesis of depression (Castrén, 2005). Hippocampus is one of the most stress-sensitive brain regions (McEwen, 2008) and hippocampus is implicated in the pathogenesis of depression and mediation of antidepressants (MacQueen and Frodl, 2011). Impaired hippocampal neurogenesis may be an important factor in precipitating episodes of depression and stim- ulation of neurogenesis may be essential for effectiveness of antidepressants (Kempermann and Kronenberg, 2003).

The effect of exercise for treating stress-related illnesses, especially depression, is well documented (Lawlor and Hopker, 2001). Physical exercise has an antidepressant action in animals and humans (Malberg et al., 2000; Santarelli et al., 2003). Manev et al. (2001) reported that antidepressant medication is closely related with hippocampal neurogenesis, and hippocampal neurogenesis appears to be necessary for the effect of antidepressants (Santarelli et al., 2003).

In addition to neurogenesis, brain-derived neurotrophic factor (BDNF) has been suggested as an important factor in the patho-
${ }^{*}$ Corresponding author: Dae-Young Kim (ib https://orcid.org/0000-0002-4662-4463 Department of Sports Healthcare, College of Humanities \& Social Sciences, Inje University, 197 Inje-ro, Gimhae 50834, Korea

Tel: +82-55-320-3171, Fax: +82-55-320-3545, E-mail: cdps21@inje.ac.kr

Received: November 5, 2017 / Accepted: December 6, 2017
This is an Open Access article distributed under the terms of the Creative Commons Attribution Non-Commercial License (http://creativecommons.org/licenses/by-nc/4.0/) which permits unrestricted non-commercial use, distribution, and reproduction in any medium, provided the original work is properly cited. 
genesis of depression or in the mechanism of antidepressants (Santarelli et al., 2003). Exercise-induced BDNF increment is associated with therapeutic effect of exercise on depression (Monteggia et al., 2004). Koponen et al. (2005) reported that BDNF knockout mice showed impaired antidepressant response and infusion of BDNF into the hippocampus produced antidepressive effect. Depression-induced reduction of neurotrophic factors, such as BDNF, decreased neurogenesis, meanwhile decreased neurotrophic factors including BDNF were restored by exercise (Redila and Christie, 2006).

Based on the previous studies, we hypothesize that treadmill exercise enhances hippocampal neurogenesis via increasing BDNF expression in social isolation-induced depression. In the present study, the effect of treadmill exercise on depression-like conditions using social isolated rat pups was investigated.

\section{MATERIALS AND METHODS}

\section{Animals and treatments}

Male and female Sprague-Dawley rat pups were used in this experiment. The experimental procedures were performed in accordance with the animal care guidelines of the National Institutes of Health and the Korean Academy of Medical Sciences. The day of delivery was designated postnatal day 0 . On the postnatal day 14 , each rat pup from each dam was divided into one of the four groups: the control group, the exercise group, the social isolation group, and the social isolation and exercise group ( $\mathrm{n}=8$ in each group). The rat pups in the control group and exercise group were housed with their respective mothers under the standard conditions, while the rat pups in the social isolation groups were housed individually. The social isolation started on the postnatal day 14 . All the rat pups were injected subcutaneously with 50-mg/kg 5-bromo-2'-deoxyuridine (BrdU) (Sigma Chemical Co., St Louis, MO, USA) every other day at $1 \mathrm{hr}$ prior to starting treadmill exercise from postnatal day 21 to postnatal day 26 .

\section{Treadmill exercise protocol}

The rat pups in the exercise groups were forced to run on treadmill for 30 min once a day from postnatal day 21 to postnatal day 34. The exercise load consisted of running at a speed of $2 \mathrm{~m} / \mathrm{min}$ for the first $5 \mathrm{~min}$, at a speed of $5 \mathrm{~m} / \mathrm{min}$ for the next $5 \mathrm{~min}$, and then at a speed of $8 \mathrm{~m} / \mathrm{min}$ for the last 20 min with $0 \%$ grade of inclination.

\section{Forced swimming test}

In order to evaluate the degree of a depression in the rat pups, we performed a modified forced swimming test, according to a previously described method (Khisti et al., 2000). On postpartum day 32, the rat pups conducted a pretest for 15 min to eliminate the acute stress by water and to adapt the animals to the water. Twenty-four hours after the pretest, the rat pups were tested for $10 \mathrm{~min}$. The animals were placed individually in the glass cylinder $20 \mathrm{~cm}$ in diameter and $90 \mathrm{~cm}$ in height, filled with water to a height of $75 \mathrm{~cm}$. The temperature of the water was adjusted to $25^{\circ} \mathrm{C} \pm 1^{\circ} \mathrm{C}$. During the test session, resting time and mobility time were analyzed using a Smart version 2.5 video tracking system (Panlab, Barcelona, Spain). Resting was defined to occur when no additional activity was observed other than the actions needed to keep the rat's head above the water. Mobility was defined to occur when the rat was in an active vertical motion with its forelegs above the water level.

\section{Tissue preparation}

To begin the sacrificial process, the animals were fully anesthetized using Zoletil $50(10 \mathrm{mg} / \mathrm{kg}$ intraperitoneally; Vibac Laboratories, Carros, France). After a complete lack of response was observed, the rat pups were transcardially perfused with $50 \mathrm{mM}$ phosphate-buffered saline (PBS) and subsequently fixed with freshly prepared $500 \mathrm{mM}$ phosphate buffer $(\mathrm{pH}, 7.4)$ containing $4 \%$ paraformaldehyde. The brains of the rat pups were removed and fixed in the same fixative overnight and then transferred into a $30 \%$ sucrose solution for cryoprotection. Serial coronal sections of $40 \mu \mathrm{m}$ thickness were obtained using a freezing microtome (Leica, Nussloch, Germany).

Immunofluorescence for 5-hydroxytryptamine and tryptophan hydroxylase expression

Immunofluorescence was conducted to evaluate the expression of 5-hydroxytryptamine (5-HT)-positive and tryptophan hydroxylase (TPH)-positive cells in the dorsal raphe, according to a previously described method (Sung et al., 2012). The sections were then incubated overnight with rabbit polyclonal anti-5-HT antibody (1:500; Immuno Star, Hudson, WI, USA) and mouse monoclonal anti-TPH antibody (1:500; Oncogene Research Products, Cambridge, UK). The sections were next incubated for $90 \mathrm{~min}$ with CY3 anti-rabbit secondary antibody (Vector Laboratories, Burlingame, CA, USA) and FITC anti-mouse secondary antibody (Jackson ImmunoResearch Laboratories, West Grove, PA, USA). The sections were then mounted on gelatin-coated glass slides, 
and the coverslips were mounted using fluorescent mounting medium (DakoCytomation, Carpinteria, CA, USA).

\section{Immunohistochemistry for BrdU}

For the detection of newly generated cells in the hippocampal dentate gyrus, BrdU immunohistochemistry was performed, according to a previously described method (Baek et al., 2012). The sections were incubated overnight at $4^{\circ} \mathrm{C}$ with mouse monoclonal anti-BrdU antibody (1:600; Roche, Mannheim, Germany). Then, the sections were washed 3 times with PBS and incubated for 90 min with the biotionylated mouse secondary antibody (1:200; Vector Laboratories). After BrdU-specific staining, counter-staining was performed on the same sections using a mouse monoclonal anti-neuronal nucleic $(\mathrm{NeuN})$ antibody (1:300; Chemicon International, Temecula, CA, USA). The slides were air dried overnight at room, and coverslips were mounted with Permount (Fisher Scientific, New Jersey, NJ, USA). The numbers of BrdU-positive cells in the dentate gyrus was counted hemilaterally under a light microscope (Olympus, Tokyo, Japan), and they were expressed as the number of cells per square millimeter in the dentate gyrus.

\section{Western blot analysis}

Western blot analysis for BDNF and tyrosine kinase B (TrkB) was performed, according to a previously described method (Kim et al., 2010). Proteins were then transferred to nitrocellulose and the blots were probed with anti-BDNF rabbit polyclonal antibody (1:1,000, Santa Cruz Biotechnology, Santa Cruz, CA, USA) or anti-TrkB rabbit polyclonal antibody $(1: 1,000$, Santa Cruz Biotechnology). Peroxidase anti-rabbit IgG (1:5,000, Vector Labora- tories) was used as the secondary antibodies. Immunoreactivity was detected by enhanced chemiluminescence detection kit (Santa Cruz Biotechnology).

\section{Data analysis}

Differences among the groups were evaluated using one-way analysis of variance followed by Duncan post hoc test. All values are expressed as the mean \pm standard error of the mean. Statistically significant differences were established at $P<0.05$.

\section{RESULTS}

\section{Effect of treadmill exercise on depression}

Forced swimming test was conducted for the evaluation of depression state (Fig. 1). The resting time for the social isolation group was longer than that for the control group $(P<0.05)$. Treadmill exercise significantly decreased the resting time of the rat pups in the social isolation group $(P<0.05)$. The mobility time for the social isolation group was shorter than that for the control group $(P<0.05)$. Treadmill exercise significantly increased the mobility time of the rat pups in the social isolation group $(P<0.05)$.

\section{Effect of treadmill exercise on the 5-HT synthesis and TPH expression}

Photomicrographs of 5-HT and TPH-positive cells in the dorsal raphe are shown in Fig. 2. The number of 5-HT-positive cells in the social isolation group was lower than that in the control group $(P<0.05)$. The number of 5-HT-positive cells in the social isolation and exercise group was higher than that in the social iso-
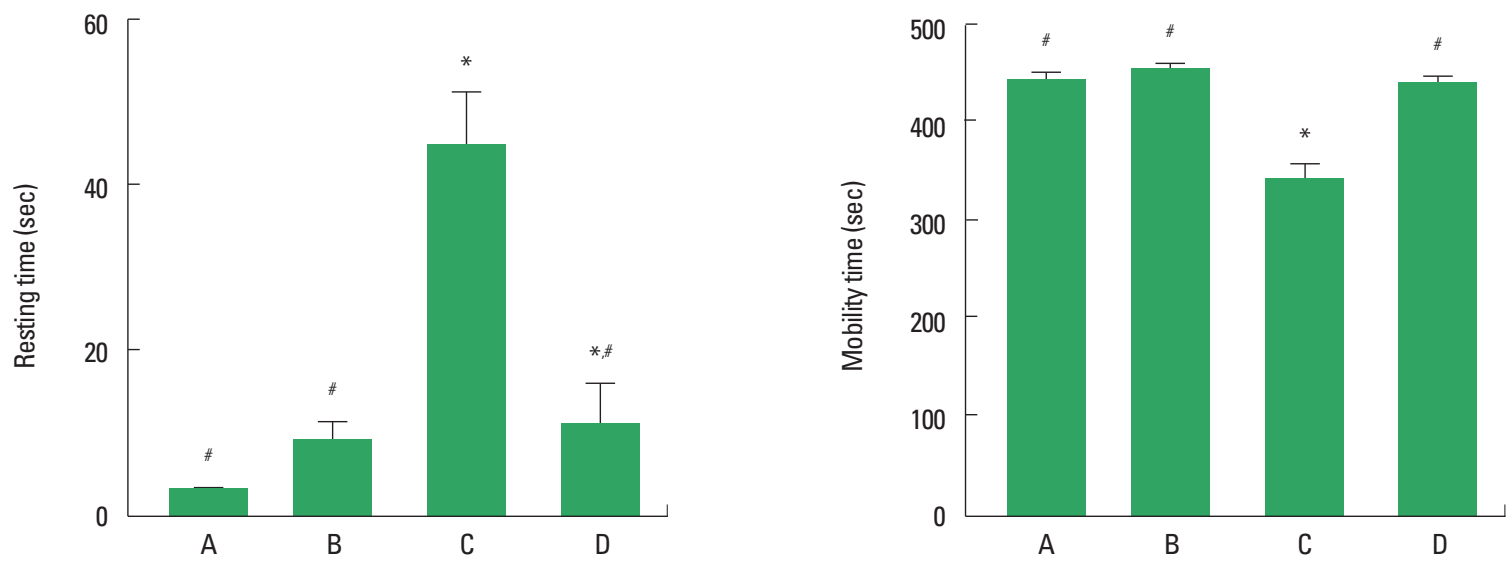

Fig. 1. Forced swimming test. Left panel: Resting time. Right panel: Mobility time. A, control group; B, exercise group; C, social isolation group; D, social isolation and exercise group. The data are represented as the mean \pm standard error of the mean. ${ }^{*} P<0.05$ compared to the control group. ${ }^{\sharp} P<0.05$ compared to the social isolation group. 

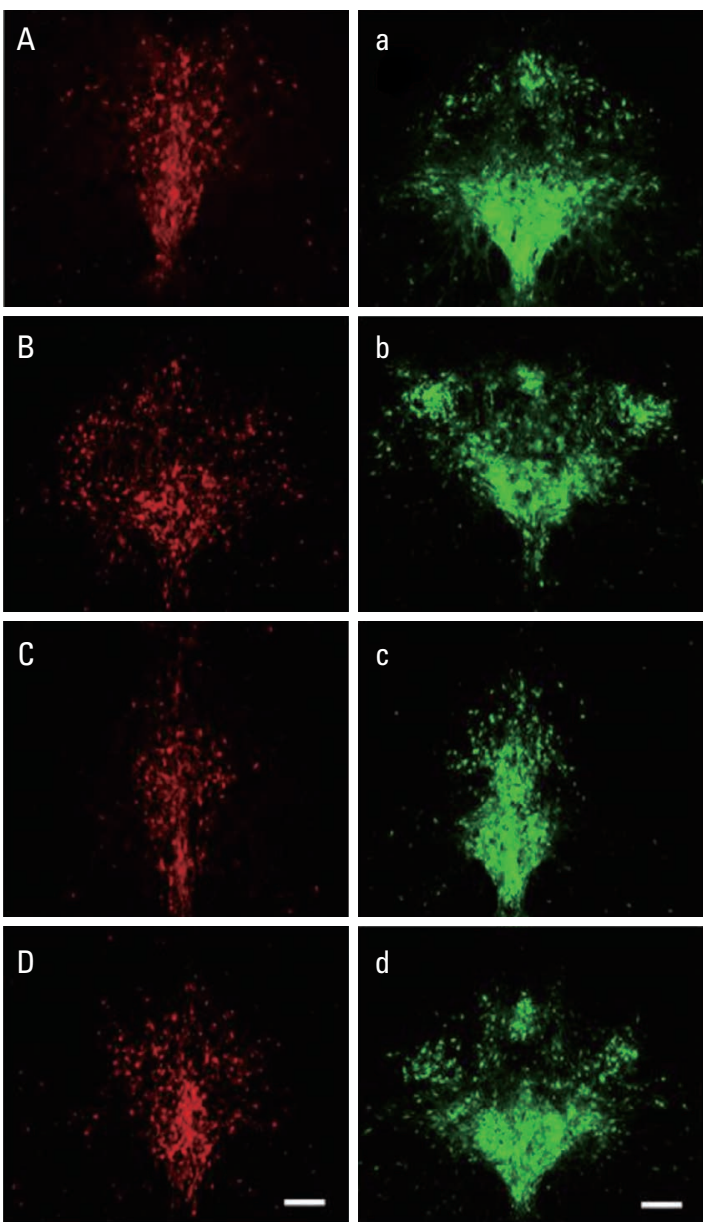
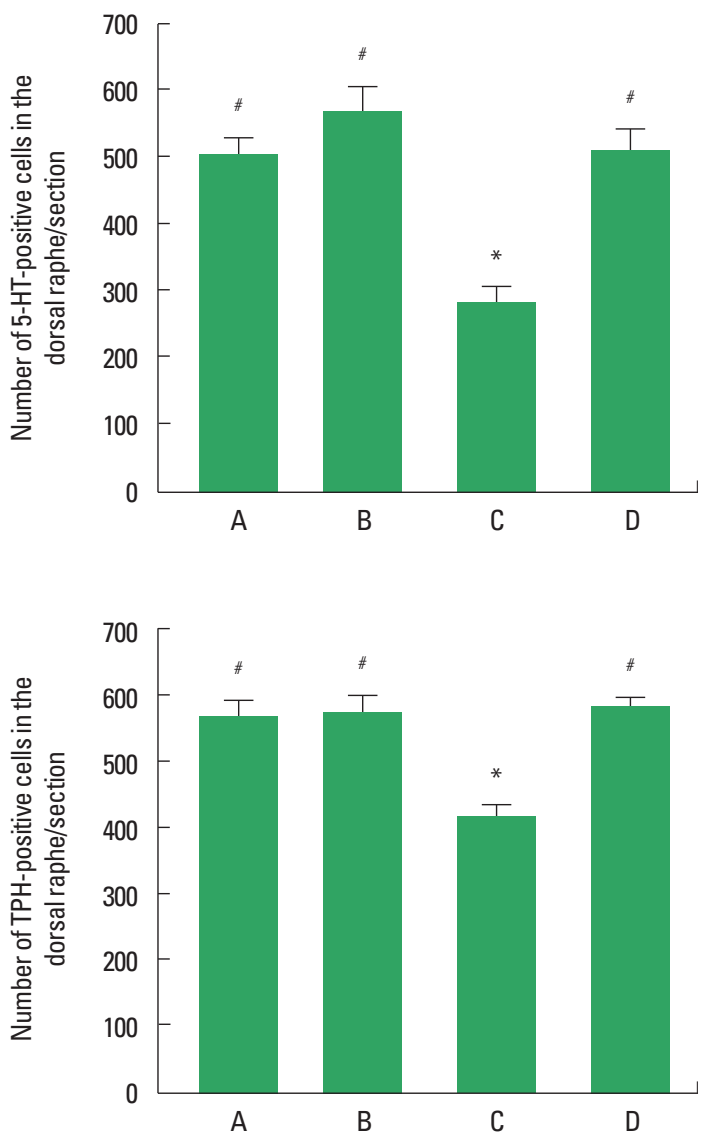

Fig. 2. 5-Hydroxytryptamine (5-HT) and tryptophan hydroxylase (TPH)-positive cells in the dorsal raphe. Left panel: Photomicrographs of 5-HT-positive and TPH-positive cells in the dorsal raphe. A and a, control group; $B$ and b, exercise group; $C$ and c, social isolation group; $D$ and d, social isolation and exercise group. The scale bar represents $200 \mu \mathrm{m}$. Right panel: Number of 5-HT-positive and TPH-positive cells in each group. A, control group; B, exercise group; C, social isolation group; D, social isolation and exercise group. The data are represented as the mean \pm standard error of the mean. ${ }^{*} P<0.05$ compared to the control group. ${ }^{\#} P<0.05$ compared to the social isolation group.

lation group $(P<0.05)$. The number of TPH-positive cells in the social isolation group was lower than that in the control group $(P<0.05)$. The number of TPH-positive cells in the social isolation and exercise group was higher than that in the social isolation group $(P<0.05)$.

\section{Effect of treadmill exercise on neurogenesis}

Photomicrographs of BrdU-positive cells in the hippocampal dentate gyrus are shown in Fig. 3. The number of BrdU-positive cells in the social isolation group was lower than that in the control group $(P<0.05)$. The number of BrdU-positive cells in the social isolation and exercise group was higher than that in the social isolation group $(P<0.05)$.

\section{Effect of treadmill exercise on BDNF and TrkB expression}

Western blot analysis for the expression of BDNF and TrkB in the hippocampus was performed (Fig. 4). The expression of BDNF and TrkB was decreased by social isolation $(P<0.05)$. Treadmill exercise increased BDNF and TrkB expression in the rat pups of social isolation group $(P<0.05)$.

\section{DISCUSSION}

Social isolation induces various neural and behavioral changes in the distressed animals (Stowe et al., 2005). In the present study, the rat pups were isolated from their peers from postnatal day 14 to postnatal day 28. Depression-like symptoms can be evaluated by forced swimming test (Dandekar et al., 2009). In the forced 

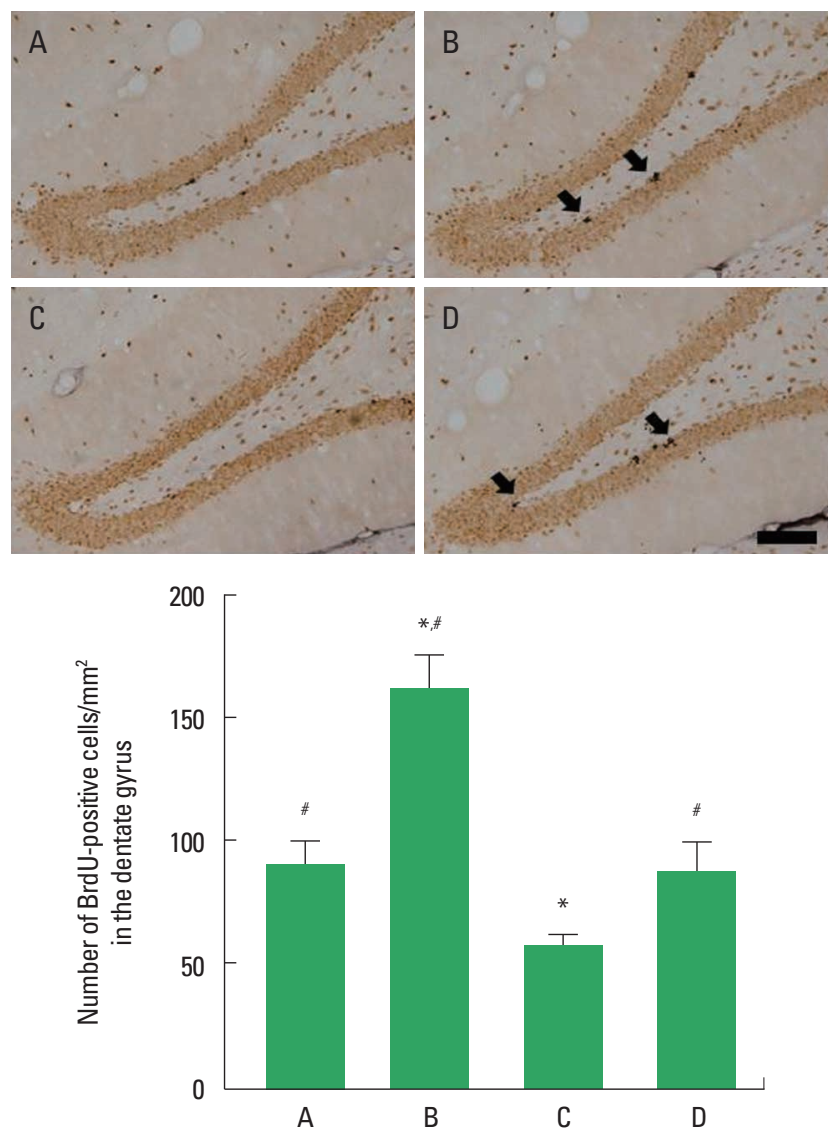

Fig. 3. Cell proliferation in the hippocampal dentate gyrus. Upper panel: Photomicrographs of 5-bromo-2'-deoxyuridine (BrdU)-positive cells. Arrows indicate BrdU-positive cells. The scale bar represents $200 \mu \mathrm{m}$. Lower panel: Number of BrdU-positive cells in each group. A, control group; $B$, exercise group; $C$, social isolation group; $D$, social isolation and exercise group. The data are represented as the mean \pm standard error of the mean. ${ }^{*} P<0.05$ compared to the control group. ${ }^{\sharp} P<0.05$ compared to the social isolation group.

swimming test, the time of resting and mobility is critical indicator determining depression state. In the present study, we found an increase in resting time and a decrease in mobility time in the rat pups of social isolation group. Treadmill exercise shortened resting time and increased mobility time. These results showed that treadmill exercise alleviated depression of the rat pups isolated from their peers.

Serotonin (5-HT) is related with depression and adult neurogenesis. Serotonergic systems in the brain are influenced by various chronic stresses (Lee et al., 2001), and decreased activity of the brain serotonergic system is implicated in the pathophysiology of depression (Arborelius et al., 2004). In order to confirm whether social isolation induced depression, we measured 5-HT synthesis and TPH expression in the dorsal raphe. In this study, 5-HT syn-

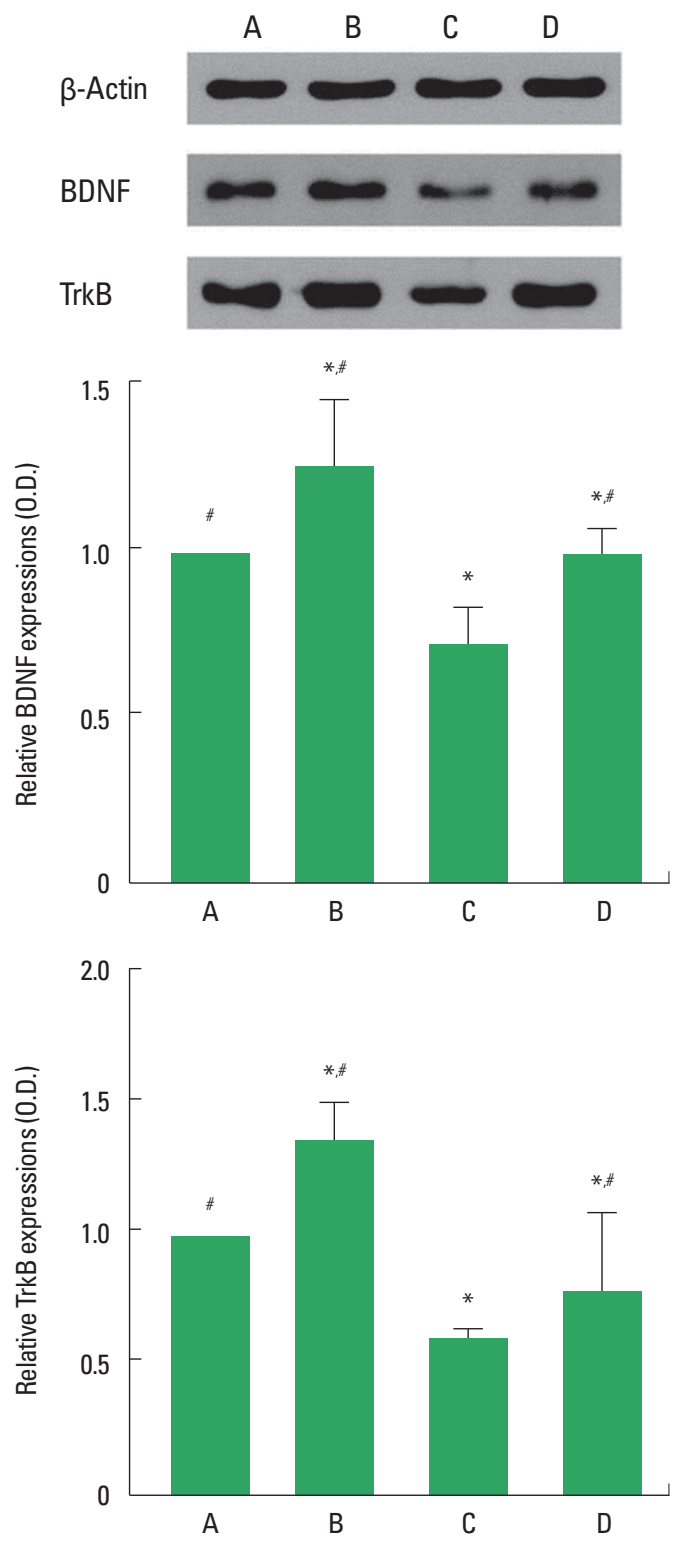

Fig. 4. Brain-derived neurotrophic factor (BDNF) and tyrosine kinase B (TrkB) expression in the hippocampus. Upper panel: Representative expression of BDNF, TrkB, and $\beta$-actin. Lower panel: Relative BDNF and TrkB expression in each group. $A$, control group; $B$, exercise group; $C$, social isolation group; $D$, social isolation and exercise group. The data are represented as the mean \pm standard error of the mean. ${ }^{*} P<0.05$ compared to the control group. ${ }^{*} P<0.05$ compared to the social isolation group.

thesis in the dorsal raphe of rat pups was suppressed by social isolation, but treadmill exercise increased the expression of 5-HT. This tendency was supported by Baek et al. (2012) showing that 5-HT synthesis in the dorsal raphe was lower in the maternal separation model than that in the maternal care rats. Yang et al. (2008) also reported that 5-HT level in the dorsal raphe was lower in the 
depression model compared to the normal rats. As TPH is the rate-limiting enzyme for the synthesis of 5-HT, reduction in TPH leads to a rapid decrease in 5-HT release (Lee et al., 2001). In this study, TPH expression in the dorsal raphe of rat pups was decreased by social isolation and treadmill exercise increased the expression of TPH. The present results suggested that treadmill exercise restored serotonin level in the dorsal raphe of social isolated rat pups.

Chronic stresses, such as social isolation, play a major role in the regulation of postnatal neurogenesis (Mirescu and Gould, 2006). Lee et al. (2001) showed that a decrement of neurogenesis was related to the pathogenesis of depression. Fabricius et al. (2008) demonstrated that stressful experiences are known to suppress neurogenesis in the hippocampal dentate gyrus. Based on previous studies, decrease in neurogenesis can be considered as a hallmark for depression. In the present study, the number of BrdU-positive cells in the hippocampal dentate gyrus of rat pups was decreased by social isolation. However, treadmill exercise significantly increased cell proliferation in the hippocampal dentate gyrus of social isolated rat pups.

Physical exercise stimulates local neurotrophic factor and enhances plasticity (Gómez-Pinilla et al., 2002). Especially, BDNF has been suggested as a principal mediator for the exercise effect on the overall brain function. Stress-induced reduction of neurotrophic factors, such as BDNF, may decrease neurogenesis (Rasmusson et al., 2002). In this study, the expression of BDNF in the hippocampus of rat pups was decreased by social isolation, but treadmill exercise increased BDNF level in the social isolated rat pups. The result of our study is relevant with previous study showing that expression of BDNF in the hippocampus of depressive animal models was decreased and that voluntary exercise restored BDNF level (Fuss et al., 2010).

Exercise is currently advocated as a behavioral intervention to increase new cell generation and survival in the hippocampal dentate gyrus (Sim et al., 2004). Increased neurogenesis in the hippocampal dentate gyrus is closely associated with ameliorating effect of depressive state (Taliaz et al., 2010). The present results suggested that treadmill exercise may ameliorates social isolation-induced depression symptoms through increasing neuronal generation.

\section{CONFLICT OF INTEREST}

No potential conflict of interest relevant to this article was reported.

\section{ACKNOWLEDGMENTS}

This work was supported by the 2016 Inje University research grant.

\section{REFERENCES}

Arborelius L, Hawks BW, Owens MJ, Plotsky PM, Nemeroff CB. Increased responsiveness of presumed 5-HT cells to citalopram in adult rats subjected to prolonged maternal separation relative to brief separation. Psychopharmacology (Berl) 2004;176:248-255.

Baek SS, Jun TW, Kim KJ, Shin MS, Kang SY, Kim CJ. Effects of postnatal treadmill exercise on apoptotic neuronal cell death and cell proliferation of maternal-separated rat pups. Brain Dev 2012;34:45-56.

Castrén E. Is mood chemistry? Nat Rev Neurosci 2005;6:241-246.

Dandekar MP, Singru PS, Kokare DM, Subhedar NK. Cocaine- and amphetamine-regulated transcript peptide plays a role in the manifestation of depression: social isolation and olfactory bulbectomy models reveal unifying principles. Neuropsychopharmacology 2009;34:12881300.

Fabricius K, Wörtwein G, Pakkenberg B. The impact of maternal separation on adult mouse behaviour and on the total neuron number in the mouse hippocampus. Brain Struct Funct 2008;212:403-416.

Fone KC, Porkess MV. Behavioural and neurochemical effects of post-weaning social isolation in rodents-relevance to developmental neuropsychiatric disorders. Neurosci Biobehav Rev 2008;32:1087-1102.

Fuss J, Ben Abdallah NM, Vogt MA, Touma C, Pacifici PG, Palme R, Witzemann V, Hellweg R, Gass P. Voluntary exercise induces anxiety-like behavior in adult C57BL/6J mice correlating with hippocampal neurogenesis. Hippocampus 2010;20:364-376.

Gómez-Pinilla F, Ying Z, Roy RR, Molteni R, Edgerton VR. Voluntary exercise induces a BDNF-mediated mechanism that promotes neuroplasticity. J Neurophysiol 2002;88:2187-2195.

Kempermann G, Kronenberg G. Depressed new neurons--adult hippocampal neurogenesis and a cellular plasticity hypothesis of major depression. Biol Psychiatry 2003;54:499-503.

Khisti RT, Chopde CT, Jain SP. Antidepressant-like effect of the neurosteroid 3alpha-hydroxy-5alpha-pregnan-20-one in mice forced swim test. Pharmacol Biochem Behav 2000;67:137-143.

Kim SE, Ko IG, Kim BK, Shin MS, Cho S, Kim CJ, Kim SH, Baek SS, Lee EK, Jee YS. Treadmill exercise prevents aging-induced failure of memory through an increase in neurogenesis and suppression of apoptosis in rat hippocampus. Exp Gerontol 2010;45:357-365.

Koponen E, Rantamäki T, Voikar V, Saarelainen T, MacDonald E, Castrén E. Enhanced BDNF signaling is associated with an antidepressant-like 
behavioral response and changes in brain monoamines. Cell Mol Neurobiol 2005;25:973-980.

Lawlor DA, Hopker SW. The effectiveness of exercise as an intervention in the management of depression: systematic review and meta-regression analysis of randomised controlled trials. BMJ 2001;322:763-767.

Lee HJ, Kim JW, Yim SV, Kim MJ, Kim SA, Kim YJ, Kim CJ, Chung JH. Fluoxetine enhances cell proliferation and prevents apoptosis in dentate gyrus of maternally separated rats. Mol Psychiatry 2001;6:610, $725-728$

MacQueen G, Frodl T. The hippocampus in major depression: evidence for the convergence of the bench and bedside in psychiatric research? Mol Psychiatry 2011;16:252-264.

Malberg JE, Eisch AJ, Nestler EJ, Duman RS. Chronic antidepressant treatment increases neurogenesis in adult rat hippocampus. J Neurosci 2000;20:9104-9110.

Manev H, Uz T, Smalheiser NR, Manev R. Antidepressants alter cell proliferation in the adult brain in vivo and in neural cultures in vitro. Eur J Pharmacol 2001;411:67-70.

McEwen BS. Central effects of stress hormones in health and disease: Understanding the protective and damaging effects of stress and stress mediators. Eur J Pharmacol 2008;583:174-185.

Mirescu C, Gould E. Stress and adult neurogenesis. Hippocampus 2006; 16:233-238.

Monteggia LM, Barrot M, Powell CM, Berton O, Galanis V, Gemelli T, Meuth S, Nagy A, Greene RW, Nestler EJ. Essential role of brain-derived neurotrophic factor in adult hippocampal function. Proc Natl Acad Sci U S A 2004;101:10827-10832.

Rasmusson AM, Shi L, Duman R. Downregulation of BDNF mRNA in the hippocampal dentate gyrus after re-exposure to cues previously associated with footshock. Neuropsychopharmacology 2002;27:133142.

Redila VA, Christie BR. Exercise-induced changes in dendritic structure and complexity in the adult hippocampal dentate gyrus. Neuroscience 2006;137:1299-1307.

Santarelli L, Saxe M, Gross C, Surget A, Battaglia F, Dulawa S, Weisstaub N, Lee J, Duman R, Arancio O, Belzung C, Hen R. Requirement of hippocampal neurogenesis for the behavioral effects of antidepressants. Science 2003;301:805-809.

Sim YJ, Kim SS, Kim JY, Shin MS, Kim CJ. Treadmill exercise improves short-term memory by suppressing ischemia-induced apoptosis of neuronal cells in gerbils. Neurosci Lett 2004;372:256-261.

Stowe JR, Liu Y, Curtis JT, Freeman ME, Wang Z. Species differences in anxiety-related responses in male prairie and meadow voles: the effects of social isolation. Physiol Behav 2005;86:369-378.

Sung YH, Kim SC, Hong HP, Park CY, Shin MS, Kim CJ, Seo JH, Kim DY, Kim DJ, Cho HJ. Treadmill exercise ameliorates dopaminergic neuronal loss through suppressing microglial activation in Parkinson's disease mice. Life Sci 2012;91:1309-1316.

Taliaz D, Stall N, Dar DE, Zangen A. Knockdown of brain-derived neurotrophic factor in specific brain sites precipitates behaviors associated with depression and reduces neurogenesis. Mol Psychiatry 2010;15:8092.

Yang RJ, Mozhui K, Karlsson RM, Cameron HA, Williams RW, Holmes A. Variation in mouse basolateral amygdala volume is associated with differences in stress reactivity and fear learning. Neuropsychopharmacology 2008;33:2595-2604. 